Der Unterschied der beiden reduzierten Potentiale $s$ liegt über dem üblichen Fehler für Hinderungspotentiale. Erstaunlich ist auch der Unterschied der Winkel $\vartheta$. Ändert man den Winkel $\vartheta$ für $v=1$ willkürlich von $70^{\circ}$ auf $58^{\circ}$, so verringert sich $s$ um etwa 0,2 , beseitigt also nicht die Differenz der beiden $s$-Werte. Die Wiedergabe der Torsionsfeinstruktur verschlechtert sich dabei. Sollten sich bei anderen Molekülen ähnliche Verhältnise zeigen, wofür Hinweise bestehen ${ }^{11}$, so sollte man alle bisher bestimmten Hinderungspotentiale bezüglich ihrer Interpretation mit größerer Vorsicht bewerten. Infolge eines mangelhaften Modells wäre es dann nicht statthaft, die Meßgenauigkeit der Aufspaltungsmessungen ohne weiteres auf die Potentialwerte zu übertragen. Ähnliche Probleme treten bekanntlich bei den Strukturbestimmungen aus Rotationsspektren auf.

Die Schwingungssatelliten fügen sich zwar gut in das Spektrum eines starren Rotators ein ${ }^{4}$, doch scheint

11 D. Sutter, Dissertation, Freiburg 1966. - D. Sutter, H. Dreizler u. H. D. Rudolph, Z. Naturforschg. 20 a, 1676 [1965].

\section{Eine Methode zur direkten Bestimmung des Leitfähigkeitsprofils in Plasmen}

\author{
H. Burkhardt und H. Rapp \\ Institut für Plasmaforschung der Technischen Hochschule \\ Stuttgart
}

(Z. Naturforschg. 21 a, 2102-2104 [1966] ; eingeg. am 22. Oktober 1966)

Die Bestimmung der elektrischen Leitfähigkeit $\sigma$ von Plasmen erfolgt in der Regel indirekt über Temperatur-, Stoßquerschnitts- und Dichtemessungen. Eine direkte Messung ohne störende Sonden ist durch den $\mathrm{Zu}$ sammenhang der elektrischen Leitfähigkeit mit der Skintiefe möglich ${ }^{1-5}$. Die im folgenden beschriebene Methode gestattet eine Bestimmung von eindimensionalen Leitfähigkeitsprofilen ${ }^{6-8}$. Wir beschränken uns auf kreiszylindrische Anordnungen mit allein radialer Veränderlichkeit und auf Objekte mit der relativen Permeabilität $\mu_{\mathrm{r}}=1$.

\section{Prinzip}

Die Impedanz $\widetilde{\vartheta}$ einer zylindrischen Luftspule ändert sich, wenn wir ein elektrisch leitfähiges Objekt in die Spule einführen. Sie ist eine komplexwertige Funktion der Frequenz $\omega$ und bei kreiszylindrischen Objekten ein Funktional des Leitfähigkeitsprofils $\sigma(r)$ :

$$
\widehat{Z}=\mathfrak{Z}(\sigma(r) ; \omega) \text {. }
$$

Die Umkehrung des funktionalen Zusammenhangs lie-

1 G. M. Giannini et al., Experiments with High Intensity Electric Discharges, Proc. Intern. Symp. High Temperature Technology, California 1959, McGraw-Hill Book Co., New York 1960.

2 P. Savic u. G. T. Boult, J. Sci. Instr. 39, 258 [1962].

3 R. A. Olson u. E. C. Lary, AIAA J. 1, 2513 [1963].

${ }^{4}$ K. V.Donskor et al., Soviet Phys.-Tech. Phys. 7, 805 [1963].

5 H. Tanaca u. M. Hagi, Japan J. Appl. Phys. 3, 335 [1964]. deshalb die Kopplung zwischen der Schwingung bei $v^{*} \approx 160 \mathrm{~cm}^{-1}$ und Torsion bei $v^{*} \approx 153 \mathrm{~cm}^{-1}$ nicht vernachlässigbar zu sein, worauf die unterschiedlichen Torsionsfeinstrukturen hinweisen.

Es ist beabsichtigt, die Auswertung in 4. Ordnung $\mathrm{zu}$ wiederholen, eine Analyse analog eines mit einem Schwingungsfreiheitsgrad erweiterten Modells nach Нгвота ${ }^{12}$ zu versuchen, die Verhältnisse bei dem Spektrum $v=2 \mathrm{zu}$ studieren und genauere Messungen der Dipolkomponenten $\mu_{\mathrm{a}}$ und $\mu_{\mathrm{b}}$ durchzuführen, worüber zu gegebener Zeit ausführlich berichtet wird.

Ich danke Herrn Dr. H. D. Rudolph und Herrn Dr. D. Sutter für Diskussionen, Herrn Dr. R. Peter und Herrn Dr. G. Herberich für die Überlassung von Rechenprogrammen, Fräulein M. HaAs für die Hilfe bei der Meß- und Auswertearbeit.

Der Deutschen Forschungsgemeinschaft und dem Fonds der Chemie danke ich für finanzielle Hilfe.

12 E. Hirota, private Mitteilung.

fert die elektrische Leitfähigkeit als Funktion des Radius und als Funktional der über der Frequenz gegebenen komplexwertigen Impedanzfunktion:

$$
\sigma=\sigma(\widetilde{\jmath}(\omega) ; r) \text {. }
$$

Der geschilderte Zusammenhang transformiert das Problem der Messung des Leitfähigkeitsprofils $\sigma(r)$ in eine Messung der Impedanz als Funktion der Frequenz $\omega$.

\subsection{Impedanz und Induktivität}

Die Impedanz einer Spule mit leitfähigem Kern setzt sich aus einem frequenzabhängigen Realteil $R$ und Imaginärteil $j \omega L$ zusammen :

$$
\widehat{\delta}=R(\omega)+j \omega L(\omega) \text {. }
$$

Es gilt allgemein für harmonische Vorgänge, daß die Impedanz eine analytische Funktion der Frequenz ist ${ }^{9}$. Realteil und Imaginärteil sind also nach der CAUCHYRiemansschen Beziehung eindeutig gekoppelt. Daraus folgt, daß für die Bestimmung der Impedanz als Funktion der Frequenz eine Messung der Induktivität als Funktion der Frequenz genügt.

Die Messung der Induktivität wird mit großer Genauigkeit durch eine Frequenzmessung möglich, wenn die Spule Teil eines selbsterregten Schwingkreises ist. Für den Zusammenhang zwischen Resonanzfrequenz $\omega_{0}$ und Induktivität $L_{0}$ gilt mit sehr guter Näherung bei jeweils konstanter Kapazität $C$ ohne Meßobjekt

$$
\omega_{0}=1 / \sqrt{L_{0} C} \text {, }
$$

6 H. Burkhardt u. H. Rapp, Bericht 1-15 (1965), Inst. f. Hochtemperaturforschung der Techn. Hochschule Stuttgart.

7 E. W. Johnson u. H. H. Johnson, Rev. Sci. Instr. 35, 1510 [1964].

8 A. D. Sтокеs, Proc. Inst. Electr. Engrs. London 112, 1583 [1965].

9 P. M. Morse u. H. Feshbach, Methods of Theoretical Physics, Part 1, McGraw-Hill Book Co., New York 1953. 
und mit Meßobjekt

$$
\omega_{x}=1 / \sqrt{L\left(\omega_{x}\right) C} .
$$

Die relative Frequenzverschiebung $\Delta \Omega$ lautet dann

$$
\Delta \Omega=\left(\omega_{x}-\omega_{0}\right) / \omega_{x}=1-\sqrt{L\left(\omega_{x}\right) / L_{0}} .
$$

Durch Änderung der Frequenz $\omega_{0}$ mittels der Kapazität $C$ erhalten wir die Frequenzverschiebung als Funktion der Frequenz selbst. Damit ist die Bestimmung der Impedanz bzw. der Induktivität als Funktion der Frequenz auf eine Messung der relativen Frequenzverschiebung als Funktion der Frequenz zurückgeführt.

\subsection{Vergleich mit Modellen}

Die rechnerische Ableitung der Profilfunktion $\sigma(r)$ aus der gemessenen Frequenzfunktion $\Delta \Omega(\omega)$ unter realen Bedingungen bereitet wegen nicht faßbarer Inhomogenitäten des Feldes in der Spule und sonstiger apparativer Parameter Schwierigkeiten. Wir lösen das Problem, indem wir den Zusammenhang $\sigma(r)$ mit $\Delta \Omega(\omega)$ durch Modellmessungen empirisch herstellen. Durch Vorgabe einer Anzahl von bekannten Profilfunktionen $\sigma_{\nu}(r)$ aus Metallpulver-Kunststoff-Gemischen oder Graphit ${ }^{10}$ erstellen wir einen Katalog von Eichkurven für die Apparatur. Die Meßkurve $\Delta \Omega(\omega)$ eines unbekannten Objekts kann in den Eichkurvenkatalog eingezeichnet und mit den Meßkurven bekannter Profile verglichen werden.

\section{Numerische Lösungen}

Lösungen der MAxwellschen Gleichungen ohne Verschiebungsstrom für eine Spule mit konzentrisch angeordnetem, leitfähigen Körper sind unter der Voraussetzung eines homogenen axialen Magnetfeldes numerisch berechnet worden. Aus ihnen gewinnen wir die re- lative Frequenzverschiebung eines Schwingkreises als Funktion der Frequenz bei vorgegebener Profilfunktion $\sigma(r)$. Parasitäre Induktivitäten und Kapazitäten sowie Einflüsse der Dämpfung auf die Frequenz sind vernachlässigt worden.

Wir haben für die Rechnung eine Anzahl von Profilen verwendet, wie sie bei Lichtbögen oder Plasmastrahlen häufig vorkommen. Sämtliche Profile sind so gewählt, daß sie den gleichen OHmschen Leitwert für den Zylinder ergeben.

In Abb. 1 stehen die Frequenzverschiebungen $\Delta \Omega(\omega)$ den jeweiligen Profilfunktionen gegenüber. Die Asymptoten der Frequenzverschiebungen ergeben sich aus dem Verhältnis der Induktivitäten mit und ohne Objekt bei vollständiger Feldverdrängung im Objekt. Sie sind nach Gl. (6) eine Funktion der Radien von Spule und Objekt :

$$
\Delta \Omega_{\max }=1-\sqrt{1-\left(R_{0} / R_{\mathrm{sp}}\right)^{2}} .
$$

Die Frequenzfunktionen sämtlicher Profile mit dem Außenradius $R_{1}$ streben daher einem höheren Grenzwert zu als die Frequenzfunktion des durch $R_{2}$ begrenzten Profils (Abb. 1). Wegen der abschirmenden Wirkung der äußeren Schichten des Objekts gegenüber dessen Inneren erfolgt eine Wichtung der Radien. Diese bewirkt, daß eine Schicht bestimmter Dicke und Leitfähigkeit in der Randzone eine wesentlich höhere Frequenzverschiebung als im Kern hervorruft. Bei Sattelprofilen (punktierte Kurve in Abb. 1) verursacht diese Tatsache eine geringe Differenzierung der Frequenzfunktionen.

Aus der Anwendung der Maxwellschen Gleichungen auf das vorliegende Problem folgt für die Induktivität, welche durch Gl. (3) definiert ist, daß Frequenz und Profilfunktion stets als Produkt auftreten. Wegen Gl. (6) gilt dies ebenso für die Frequenzverschiebung. Es ergibt sich daraus für die Profilfunktion und die Frequenzfunktion ein Ähnlichkeitsgesetz. Danach er-
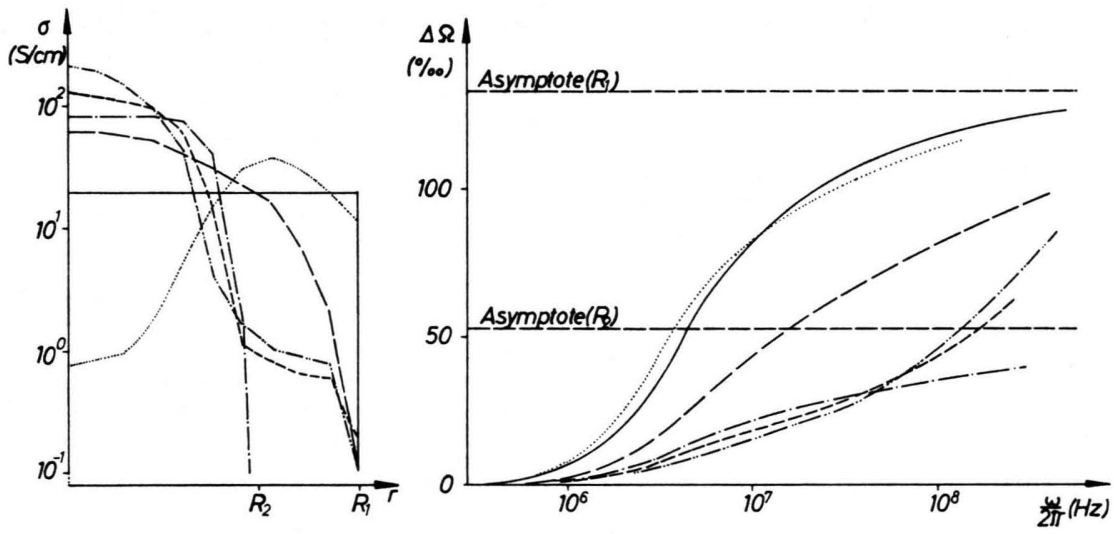

Abb. 1. Berechnete Frequenzfunktionen auf Grund vorgegebener Profilfunktionen.

10 H. Burkhardt u. K. Hirsch, Bericht 1-17 (1965), Inst. f. Hochtemperaturforschung der Techn. Hochschule Stuttgart. 
geben sämtliche Profile, welche der Beziehung

$$
\sigma_{\nu}=\sigma_{0} \cdot f_{\nu}(r)
$$

gehorchen, Frequenzkurven der Gestalt

$$
\Delta \Omega_{v}=g_{v}\left(\omega / \omega_{0}\right),
$$

wobei für die normierenden Größen der Zusammenhang gilt:

$$
\omega_{0} \sigma_{0}=\text { const . }
$$

Bei affiner Abbildung des Leitfähigkeitsprofils in $\sigma$ Richtung wird die zugehörige Frequenzfunktion entsprechend Gl. (10) ebenfalls affin abgebildet.

\section{Messung von Eichobjekten}

Die hier beschriebenen Messungen sind an zweistufigen Modellen aus Graphit durchgeführt worden, mit

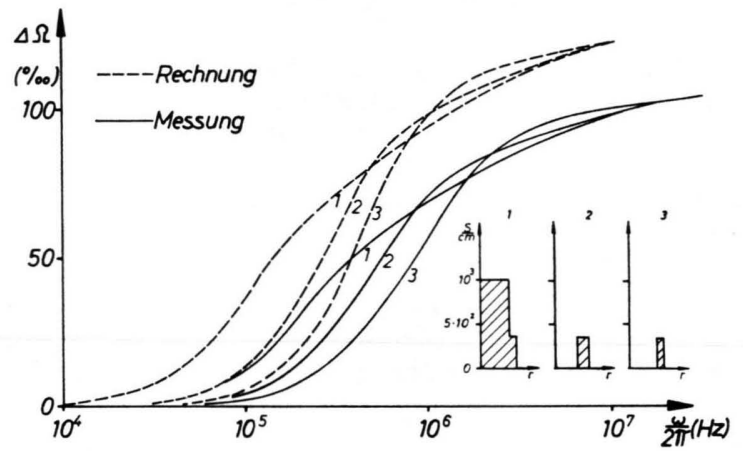

Abb. 2. Rechen- und Meßwerte verschiedener Leitfähigkeitsprofile. denen der Verlauf einiger Profiltypen grob approximiert werden kann. Der Oszillator ist als kapazitive Dreipunktschaltung ausgeführt. Der Frequenzbereich reicht von $50 \mathrm{kHz}$ bis $30 \mathrm{MHz}$. Die Feinverstimmung der Frequenz wird durch Zuschalten von Kapazitäten erreicht, während die Grobeinstellung durch die Wahl geeignet bemessener Spulen geschieht. Die Meßgenauigkeit der Anordnung liegt unter 1\%oo bezüglich der Reproduzierbarkeit. Bei Vergleichsmessungen ist allein die Kurzzeitstabilität des Oszillators entscheidend. Sie ist besser als $10^{-4}$

Die gezeigten Meßkurven sind der Übersicht wegen so korrigiert, daß ein stetiger Kurvenzug entsteht. Beim Wechsel der Spulen ändern sich nämlich gewisse Schaltungsparameter, so daß an den Spulen-Bereichs-Übergängen Unstetigkeiten im Meßkurvenverlauf auftreten. Alle Kurven laufen entsprechend den gleichen Randleitfähigkeiten und Abmessungen der Modelle bei etwa $10 \mathrm{MHz}$ zusammen. Die Gegenüberstellung der Meßkurven mit den errechneten Werten ergibt qualitativ denselben Verlauf. Die Meßergebnisse liegen jedoch unter den Rechenwerten, was entsprechend den idealisierten Voraussetzungen der Rechnung zu erwarten ist.

Das beschriebene Meßverfahren bietet die Möglichkeit der Messung von Leitfähigkeitsprofilen ohne Störung des Objekts. Die Messung bleibt durch fremde magnetische und elektrische Felder weitgehend unbeeinflußt. Die Messung und Kontrolle einzelner Kennwerte ist kontinuierlich möglich. Für die Anwendung an ebenen Objekten kann die Anordnung abgewandelt werden.

Wir danken Herrn Prof. Dr. KLuge für die Förderung dieser Arbeit.

\section{Das paramagnetische Moment eines stromdurchflossenen Zylinders in einem longitudinalen Magnetfeld}

\author{
H. Bross * und R. JAGGI ** \\ (Z. Naturforschg. 21 a, 2104-2105 [1966] ; eingeg. am 9. November 1966)
}

The paramagnetic moment of a current-carrying cylinder in a longitudinal magnetic field is explained phenomenologically. Expressions in terms of the galvanomagnetic coefficients are given for three examples: cubic crystal, trigonal crystal, two-band model with spherical energy-surfaces and energyindependent relaxation times.

Vor kurzem wurde von einem von uns $^{1}$ ein paramagnetisches Moment an einem zylindrischen WismutEinkristall beobachtet, durch den ein Strom $J$ hindurchgeschickt wurde und der sich außerdem in einem konstanten longitudinalen Magnetfeld $H$ befand. Zur Erklärung dieser Erscheinung wurde die Bewegung der Elektronen und Löcher in den äußeren Feldern sowie im Eigenmagnetfeld des Stromes untersucht. In dieser

\footnotetext{
* Sektion Physik der Universität München.

** IMB Forschungslaboratorium, 8803 Rüschlikon, Schweiz.
}

Mitteilung soll angedeutet werden, wie sich die Erscheinung völlig phänomenologisch mit den Maxwellschen Gleichungen deuten läßt, wenn man nur die durch die Magnetfelder verursachte Änderung des Tensors der elektrischen Leitfähigkeit berücksichtigt.

Der Einfachheit halber nehmen wir im folgenden an, daß das Eigenmagnetfeld

$$
H_{\varphi}=\frac{2 r}{c a^{2}} \cdot J
$$

( $a$ : Zylinderradius) des äußeren Stromes wesentlich kleiner als das äußere Magnetfeld $H$ ist, so daß wir Glieder der Art $H_{\varphi}$ gegen $H$ vernachlässigen dürfen. Weiter wollen wir von jenem Magnetfeld absehen, das mit der azimutalen Stromdichte $j_{\varphi}$ verknüpft ist. Durch den obigen Ansatz für das Magnetfeld wird dann die erste Hälfte der Maxwellschen Gleichungen identisch befriedigt. Aus der Forderung, daß das $\boldsymbol{E}$-Feld wirbelfrei sein soll, folgt weiter, $d a ß$ das Kreisintegral $\oint E_{\varphi} \mathrm{d} \varphi$ verschwinden muß, was in einem Kristall hoher Symmetrie und entsprechender Lage des äuße-

1 R. JAGGI, Naturwiss. 53, 104 [1966]. 